\title{
Orléans - Îlot de la Charpenterie
}

$\mathrm{n}^{\circ} 068603$

\section{Thierry Massat}

\section{(2) OpenEdition \\ 12 Journals}

Édition électronique

URL : http://journals.openedition.org/adlfi/13866

ISSN : 2114-0502

Éditeur

Ministère de la culture

Référence électronique

Thierry Massat, «Orléans - Îlot de la Charpenterie », ADLFI. Archéologie de la France - Informations [En ligne], Centre, mis en ligne le 19 novembre 2014, consulté le 02 mai 2019. URL : http:// journals.openedition.org/adlfi/13866

Ce document a été généré automatiquement le 2 mai 2019.

(c) Ministère de la Culture et de la Communication, CNRS 


\title{
Orléans - Îlot de la Charpenterie
}

\author{
$n^{\circ} 068603$
}

\section{Thierry Massat}

Lien Atlas (MCC) :

http://atlas.patrimoines.culture.fr/atlas/trunk/index.php?

ap_theme=DOM_2.01.02\&ap_bbox=1.874;47.813;1.949;47.933

1 L'opération de fouille archéologique de l'îlot de la Charpenterie à Orléans a été réalisée à la demande du Service régional de l'Archéologie, avec le concours de la ville d'Orléans, à partir du 21 juillet 1997 et pour une durée de 11 mois sur le terrain. Cette intervention a porté sur une superficie d'environ $2000 \mathrm{~m}^{2}$ dans le cœur historique de la ville, à proximité de la Loire. Les données issues de cette fouille seront présentées dans le bilan scientifique 1998. En revanche, les principaux acquis de l'étude archéologique et historique préliminaire, réalisée en amont par Michel Philippe et Viviane Aubourg (Aubourg-Josset, Philippe, 1997) peuvent être abordés ici.

2 Lentement, à partir $\mathrm{du} \mathrm{XI}^{\mathrm{e}}$ s., le quartier se bâtit. Dans un premier temps, les constructions se cantonnent le long des rues de la Charpenterie, des Halles, de la Poterne et de l'Empereur. Progressivement, au cours des $\mathrm{XII}^{\mathrm{e}}$ et $\mathrm{XIII}^{\mathrm{e}}$ s., des bâtiments prennent place au centre des îlots, poussant à la création de venelles et d'une rue traversante, la rue Croche-Meffroy. Parallèlement à la mise en place du tissu urbain, qui ne variera d'ailleurs plus dans les siècles suivants, la muraille du Bas-Empire est reprise. Un port est également mentionné au pied de la Charpenterie : est-il de création médiévale ou plus ancienne ? Aucun élément ne permet de trancher. Au XIV ${ }^{\mathrm{e}}$ s., la mutation est achevée, l'ensemble du quartier est bâti et le réseau viaire est en place.

3 Les parcelles, relativement régulières, peut-être issues de l'Antiquité, sont partagées entre quelques propriétaires ecclésiastiques, quelques bourgeois et le duc d'Orléans. La localisation des propriétés de ce dernier, sur les marges, trahit peut-être son antériorité sur place. On sait par ailleurs que les $\mathrm{XIII}^{\mathrm{e}}$ et $\mathrm{XIV}^{\mathrm{e}} \mathrm{s}$. correspondent à d'importantes campagnes d'acquisition de terrains par les domaines religieux. Si les sources sont lacunaires pour le début de la période, elles permettent, à partir des $\mathrm{XIV}^{\mathrm{e}}$ et $\mathrm{XV}^{\mathrm{e}} \mathrm{s}$., de 
restituer la distribution sociale du quartier. Il s'agit d'un secteur essentiellement industrieux: certaines rues correspondent aux activités professionnelles (rue de la Charpenterie, rue de la Corroierie) ; l'activité liée au travail du bois n'est plus mentionnée dans les sources postérieures au $\mathrm{XIV}^{\mathrm{e}} \mathrm{s}$; ; les métiers de peaux (tanneurs, corroyers, mégissiers) sont les activités dominantes du quartier à partir $\mathrm{du} \mathrm{Xv}^{\mathrm{e}} \mathrm{s}$.

4 L'impact de la Guerre de Cent Ans sur la ville n'est pas à minimiser. Dans le quartier, outre l'effet direct, et somme tout léger des combats (un bombardement semble avoir atteint la rue de la Charpenterie en 1428), plusieurs maisons jugées insalubres sont rasées en prévision du siège, pour empêcher toute propagation d'incendie. L'essor des métiers de peaux attire une population de commerçants et de marchands, ce qui entraîne une modification dans la composition sociale du quartier. Une population noble et bourgeoise s'installe au nord, le long de la rue de la Charpenterie, le sud est dévolu aux activités de tannerie, et des boutiquiers et d'autres artisans prennent place entre ces deux pôles. «Le bâti médiéval parait épouser la répartition sociale du quartier, à savoir des constructions bourgeoises, complexes, sur le haut du quartier, un bâti artisanal, sur le bas, et des maisons d'architecture plus modestes entre les deux. » (Aubourg-Josset, Philippe 1997 : 101).

Lors des époques moderne et contemporaine, l'histoire du quartier reste intimement liée à celle des métiers de peaux. Après une période faste lors de la Renaissance, les $\mathrm{XVII}^{\mathrm{e}}$ et $\mathrm{xVIII}^{\mathrm{e}} \mathrm{s}$. voient cette activité décliner, notamment en raison de l'essor des filatures. Ce lent déclin se traduit dans les faits par une progressive désaffection de la population bourgeoise et commerçante du quartier. Le milieu du XIX ${ }^{e}$ s. et l'arrêt du commerce fluvial sonnent le glas des dernières tanneries, entraînant une paupérisation du quartier. A l'aube du $\mathrm{Xx}^{\mathrm{e}} \mathrm{s}$., il semble devenu un ghetto, fait de ruelles étroites et de maisons vétustes. Plusieurs tentatives municipales auront pour objectif de le dynamiser, et c'est dans ce but que le quartier est rasé en 1968 pour laisser place à un marché et un parking semi-excavé en 1971.

6 Déjà à l'occasion de ces travaux, deux campagnes de fouille ont été réalisées par une équipe placée sous la direction d'Alain Ferdière, pendant trois semaines en 1969. Deux secteurs distincts ont été choisis : l'un, au sud, a permis de mettre au jour un tronçon de l'enceinte du Bas-Empire, le second, plus au nord, se situe dans l'emprise de l'opération de fouille de 1997-98. Faute de temps et de moyens, les résultats de cette première campagne restent limités, puisque les vestiges antiques n'ont pas été atteints, mais l'on peut reprendre les trois points forts de la conclusion du rapport (Ferdière 1969 : 9) :

- «Tous les remblais, même récents, contiennent en abondance du mobilier d'époque romaine et essentiellement gallo-romaine précoce ( $\mathrm{e}^{\mathrm{er}} \mathrm{s}$. av. J.-C. - début $\mathrm{I}^{\mathrm{er}} \mathrm{s}$. apr. J.-C.) ;

- «L'habitat du haut Moyen Âge est extrêmement dense, comme le montrent les fosses du chantier nord et les éléments nombreux de céramiques mérovingiennes et carolingiennes découverts çà et là »;

- «Une grande campagne de construction semble également être attestée au XVI ${ }^{\mathrm{e}} \mathrm{s}$. par les nombreuses couches de cette époque découvertes, ce qui correspond historiquement à un regain important de l'activité économique de la ville. »

7 Par ailleurs, deux séries d'observations ont été effectuées en 1971 lors des terrassements du site (Puchal-Gellida 1973, Debal 1992), qui portaient toutes deux sur la paroi nord du site, le long de la rue de la Charpenterie. Elles mettent en évidence les $6 \mathrm{~m}$ de stratigraphie détruits lors de ces terrassements. De façon prémonitoire, A. Ferdière dans son rapport décrivait l'opération qu'il venait de réaliser de la façon suivante «Il ne 
pouvait s'agir que de sondages préliminaires, d'une toute première exploration du site (prémices) d'une opération ultérieure de beaucoup plus grande ampleur.»

8 En conclusion, nous devons insister sur le fait que l'association entre une étude historique préliminaire, la réalisation de carottages et une relecture des fouilles anciennes présente, en milieu urbain, un avantage certain par rapport à une évaluation par sondages. En effet, outre la destruction des relations stratigraphiques déjà fortement malmenées par la construction du parking, ce type de reconnaissance aurait donné une vision trompeuse du site, car réalisés dans certains secteurs, les sondages auraient traduit l'image d'un gisement entièrement détruit par les terrassements de 1971 et les constructions médiévales. Inversement, dans d'autres secteurs, ils auraient mis en évidence une stratigraphie complexe et puissante et peu perturbée.

INDEX

Index géographique : Centre, Loiret (45), Orléans

operation Sauvetage programmé (SP)

Index chronologique : Gallo-romain, Moyen Âge 